This is an electronic reprint of the original article. This reprint may differ from the original in pagination and typographic detail.

Author(s): Hakala, Ismo; Härmänmaa, Tuomo; Laine, Sanna

Title: Integrated Virtual CiNetCampus Studies Environment

Year: $\quad 2016$

Version:

Please cite the original version:

Hakala, I., Härmänmaa, T., \& Laine, S. (2016). Integrated Virtual CiNetCampus Studies Environment. In L. G. Chova, A. L. Martínez, \& I. C. Torres (Eds.), EDULEARN16 Proceedings. 8th International Conference on Education and New Learning Technologies (pp. 1297-1306). IATED Academy. EDULEARN proceedings. https://doi.org/10.21125/edulearn.2016.1264

All material supplied via JYX is protected by copyright and other intellectual property rights, and duplication or sale of all or part of any of the repository collections is not permitted, except that material may be duplicated by you for your research use or educational purposes in electronic or print form. You must obtain permission for any other use. Electronic or print copies may not be offered, whether for sale or otherwise to anyone who is not an authorised user. 


\title{
INTEGRATED VIRTUAL CINETCAMPUS STUDIES ENVIRONMENT
}

\author{
Ismo Hakala, Tuomo Härmänmaa, Sanna Laine \\ University of Jyväskylä (FINLAND)
}

\begin{abstract}
Rapid growth of data communications and technologies has created favorable conditions for a strong increase in the number of videos produced for education especially in higher education institutes. New technologies make the production of videos suitable for teaching very easy. With the help of videos, it is possible to improve flexibility and diversity in teaching. On the other hand, the management and distribution of videos has often been realized separately from other applications (e.g., learning management systems) which are used in teaching. This makes their full utilization difficult and creates extra work. This article introduces the CiNetCampus environment designed for teaching with videos. A learning management system and a video content management system form its base. Integrated to other applications supporting teaching, these form a package that appears as a unitary entity to students. At the end of this article, the role of the environment comes under the limelight in connection with our education program.
\end{abstract}

Keywords: Video lecture, virtual learning environment, video content management, blended learning

\section{INTRODUCTION}

During the past decade, fast development of technology and rapid increase in data communications have created favorable conditions for growing use of learning technologies in universities. The change in the role of technology in teaching is well demonstrated in the growth of popularity of the blended model in that area. There are many different definitions for learning in accordance with the blended model (see e.g., [1], [2]). It is generally agreed that blended learning combines face-to-face and distance learning methods. Streaming video technology, which is widely utilized in connection with distance learning, provides good opportunities to transform face-to-face learning along the lines of the blended model. Videos present an effortless and cost-efficient way to bring flexible solutions, regarding time and place, to learning.

They can be used in a form of short video clips to support face-to-face learning; however, more and more often entire courses are offered as lecture sets on video, as video lectures, together with face-toface learning, or as their replacement. Typically, video lectures aim to improve access to teaching by bringing flexibility to study. Live videos bring flexibility in relation to location, whereas on-demand videos allow the students follow teaching in a flexible manner as far as time and place are concerned. Improvement in the access to teaching and flexibility in relation to time and place enable for example participation in overlapping courses, substitution of occasional absences, combination of work and study, distance learning, etc. Video lectures also provide excellent opportunities for the revision of difficult lecture topics and for exam preparations.

On the other hand, in addition to the improvement in the access to teaching and increase in flexibility, video lectures also generate numerous challenges, making their wider implementation in education programs more difficult. The most common challenges are related to video production and implementation of interaction channels. Before the actual use of lecture videos, the education organizer needs to find solutions for the recording of those videos, for their distribution and for the management of user rights. Video lectures transform some of the students into distance students, As a result, the factors related to interaction will give rise to challenges. Especially when the role of video lectures is on the increase, it is necessary to be able to offer the students synchronous interaction forums which make communication between the students and the lecturer possible.

Thus, increasing accessibility and flexibility of education with the help of lecture videos poses also challenges. Technology and technology-based practices are at the center of finding solutions for these. However, it is very difficult to find a single application that could solve all the challenges presented. Our solution is to integrate different applications together. The core of our virtual CiNetCampus Studies learning environment consists of a commercial learning management system (LMS) and a www-based open-source-code video content management system (Video CMS). The role of LMS is to provide an easy access to written materials, tools for asynchronous communication 
and material dissemination, student management, and other services typical for LSMs. Video CMS provides a flexible way to share both live and on-demand lecture videos and synchronous communication channels, and it works also as a platform for new applications. All the applications are integrated into a seamless whole so that the environment appears as a unitary system to the students and lecturers. The design of the CiNetCampus Studies environment is based on our vision of a blended educational model that adapts to the needs of different kinds of students. For example, the students have the freedom to select, for each lecture, a participation mode that is the most suitable for them at the time. This means that, the student, not a lecturer nor a system, defines the degree of blending for him/herself. Here the degree of blending refers to the importance of the role that technological solutions have from the student's perspective.

The main contribution of this paper is its description of the current state of the virtual CiNetCampus Studies in connection with the used blended learning environment. Our blended educational model is based on face-to-face learning and strong utilization of lecture videos as well as on the practices formed around them. The educational model enables students freely choose the mode to access the education provided. CiNetCampus Studies provides the necessary support for our educational model. The paper presents the core of CiNetCampus Studies and various educational applications integrated into it.

\section{CHALLENGES RELATED TO EDUCATION BASED ON VIDEO LECTURES}

Utilization of videos in teaching requires that the education provider has solutions for video production processes and interaction. The essential parts in the video production process are video recording, post-editing, storage, distribution and management of user rights. Streaming videos, by nature, are one-way; that is, sound and image move only in one direction without a return channel. This one-way nature of streaming videos often also means that only the lecture material and possibly also the lecturer are visible to the viewers of the video. The solutions related to video production, management and interaction are of technological nature, which often creates problems in the education environment.

Today, recording of an entire lecture can be easily done with the lecturer's own computer or for example with the aid of other staff in a studio. In many cases, the lecture to be recorded will also be offered in the form of face-to-face learning. In that case, its recording in the lecture situation itself is an attractive option. Lecture events can be recorded by a person or in a fully automated manner without an assistant. Some automatic commercial recording systems may place some limitations for the materials used by the lecturer. On the other hand, automatic recording can also be implemented in such a way that the material presented from a computer or a document camera will become recorded. Post-editing of the produced video material typically remains quite minimal in educational use. This is due to the laboriousness and slowness of the process.

Videos are usually stored in the educational establishment's own or cloud-based media server, which then makes them accessible to students. It is the format of the stored video that largely determines the type of the terminal allowing the viewing of the video. Often, during the course of storing, lecture videos must still be transcoded and transrated into several formats and bitrates to make them viewable to as large extent as possible with clients using different operating systems and data communications of various speeds,

Distribution of videos is implemented generally by creating links to video files on the video server. Students can access the video links often on separate course sites on the web or on a learning management system. This typically requires that the organizer creates the links manually. Manual linking is laborious and error-prone. When a separate video content management system similar to YouTube and automatic recording are used, an automatic linking system for videos is easy to implement. In addition to YouTube, video content management systems, both commercial and those based on open source, are available. In connection with video distribution, also the user rights for the videos are considered: that is, can anyone make use of the videos or are they for the students only or for a limited group of students? Only very few video management systems provide adequate user management support.

In addition to video recording, distribution and management, video lectures bring with them teaching challenges. Regarded as some of the biggest challenges are the factors related to interaction and communication. These factors include making the students visible to each other and the interaction channels themselves. Streaming videos have a distinctive one-way character. This means that both 
image and sound are transmitted only in one direction without a return channel. To compensate for the lack of a communication channel in lecture videos, text-based chats have been used. In most of the cases, these have been found to function well. For short questions and comments, text-based chat is a natural medium. Students belonging to the mobile generation are used to chat-based communication with popular mobile applications. Nevertheless, text-based chat is not a natural medium for example for dealing with difficult matters requiring lengthy discussion or for seminars. Attempts have been made to improve opportunities for interaction by substituting real-time streaming lectures with Skype-type video call and conferencing applications such as Adobe Connect which enable real-time video conferencing among several users. Applications that enable video calls also make those attending as distance students visible to the lecturer and other students. With real-time streaming videos, the lecturer normally will not be aware of the students attending from distance. The problem with these solutions is that typically they are completely isolated from other applications used for learning and teaching. This decreases the usability of these kinds of solutions from the viewpoints of both the student and the education provider.

As mentioned earlier, there are individual solutions, both commercial and based on open source, for most of the challenges presented. Virtual learning management systems provide, among other things, a natural video link setting, student management and asynchronous interaction channel. On the other hand, the support provided by virtual learning management systems for the implementation of video clips or video lectures is usually deficient and requires the use of missing tools in parallel or their integration. The situation is similar with the video content management systems. They give good support for video management and presentation, but often their problem is lack of user management and lack of interaction channels. Due to the lack of a comprehensive solution, often the practical solution is parallel use of various tools. It is a poor option regarding usability, and it also increases work load. A better solution, as far as usability and cost-efficiency are concerned, can be achieved by integrating various applications to function as a single entity. An integrated solution also provides later opportunities to add features that are essential from the perspective of the organizer. Our approach to the development of the CiNetCampus Studies environment has been to integrate existing solutions into an integral whole from the viewpoint of the user.

\section{CINETCAMPUS STUDIES}

Our virtual CiNetCampus Studies environment consists of several applications, each serving a certain purpose in the education. The applications are integrated into a seamless whole so that the environment appears as a unitary system to the user. User management of these applications is also integrated into one place. The core of the virtual CiNetCampus Studies environment consists of a commercial learning management system (LMS) and a custom-build www-based video content management system (Video CMS).

\subsection{The Core of the CiNetCampus Studies}

Optima, a commercial LMS, plays an important role in the CiNetCampus environment. It provides an easy access to written study materials, to tools for targeted asynchronous communication and material dissemination, to student management as well as to other services typical for LMSs. The integration of LMS as a part of the environment has made the centralized management of user rights possible. From the start, it was clear that user management should be carried out only in LMS, since the students are used to constantly employing it in their studies. The students always first logon to the LMS, which has a link to the video content management system on its toolbar. A user clicking that link will be redirected from LMS to Video CMS where all the other applications of the environment can be used. The link contains a list of courses the student has enrolled in. Video CMS reads this information and updates the user privileges of the student. With the help of common user rights, it is possible to provide student-specific information or material, for example to control the student's access only to those lecture videos of the study course into which the student has been enrolled. The common user management of the environment has also significantly reduced the amount of work.

In 2010, a project was started by us in order to develop a video content management system, the second part of the core of the CiNetCampus Studies environment. We made comparisons between six software products, and finally an open-source software called ClipBucket was selected. Initially, the aim of the development of the environment was to improve video distribution in a controlled manner and interactivity among students and lecturers. Before this, we had no content management for videos. The links to videos were manually added to the LMS. The Video CMS developed is derived 
from a "YouTube clone" called ClipBucket. As the term "YouTube clone" suggests, the original version of ClipBucket has basically the same features as YouTube. Our version of ClipBucket is heavily modified and enhanced, and thus it can be classified as a derived work.

With the help of Video CMS, management of videos has become very easy. Its Video Sharing Module is the core function of the system. It provides a flexible way to share both live and on-demand lecture videos. Live videos are pre-set to be published automatically when the related face-to-face session begins. On-demand videos are automatically published once the session has ended. Videos can be accessed with students' computers and mobile devices through an Internet connection. The module also features a built-in text chat as a synchronous communication channel. There is no need to manage users and their privileges in Video CMS because that information is automatically transferred from LMS.

\subsection{The Modules of the CiNetCampus Studies}

Video CMS works also as a main platform for new modules, as illustrated in Fig. 1. The solution makes it possible to easily add new applications to the environment. In addition to the video distribution application and text chat, a commercial video chat application, an application for student participation data, an application mapping students' learning styles, an online survey design system, and, most recently, a video quality evaluation tool have been integrated into the environment. All these applications are integrated into a seamless whole so that the environment appears as a unitary system to the user.

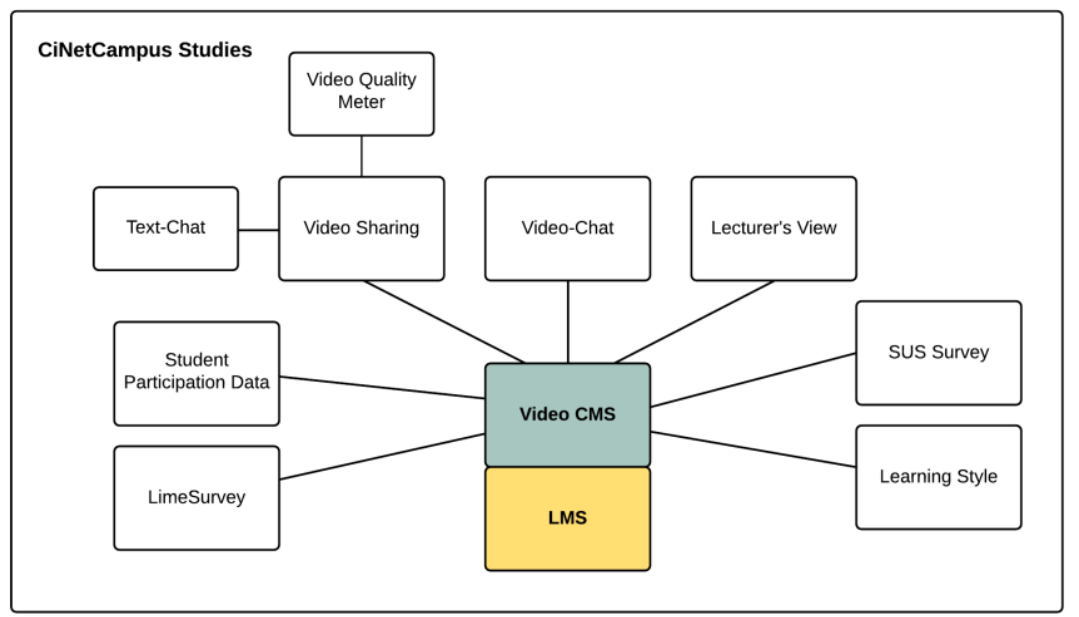

Figure 1. Modules of Video CMS

Text-Chat Module is available in all of our videos. At the left margin of the video itself, there is a list of all distance learners watching the video and a text based chat. Both the list and the chat are available when the video is played. During live sessions, distance students can use the chat to ask questions. When a chat message is sent, the lecturer can see it instantly on the lecturer's view and then respond to it. Because the text-based chat is also available in on-demand videos, students who watch the same on-demand or live video can also use the chat to communicate with each other.

Lecturer's View Module is used in the lecture room during face-to-face sessions. When the lecturer begins a session, he navigates to the front page of Video CMS where a link to the tool can be found. During the lecture session, every participant's image and name are visible in the Lecturer's View. Lecturer's View is also projected to the back wall of the teaching area. Also a text chat is included. The text chat is the default communication channel between distance learners and those in the classroom. When a student sends a chat message, the back of the lecturer's view tool starts blinking in bright color. This guarantees that no one in the classroom will miss a message from a distance learner and that the lecturer can respond instantly. Lecturers can also launch the Video-Chat Module from the Lecturer's View. After the chat is launched, distance learners who are watching the live stream are notified of this and they are able to join the Video-Chat.

Video-Chat Module is one of the most recent modules in the environment. The module was integrated into Video CMS in 2015. Video-Chat is actually a browser plugin called VidyoWeb, which has been 
developed by a company called Vidyo and which is very easy to use. There is no need to configure the microphone or webcam needed for the use of Video-Chat. Everything is done automatically. By default, each participant video feed is visible, but you can choose to make changes to the layout of the videos. If for example a participant is giving a presentation, you can make that video feed bigger. Screen sharing is an important function in this module. You can share your whole screen or just one program with others.

Video-Chat is used in face-to-face sessions, for example when distance learners need to give oral representations or participate in conversation. The use of Video-Chat isn't just limited to face-to-face sessions. The application can also be used for supervising, tutoring and group work. Students simply navigate to the Video-Chat page in Video CMS and select the room they want to join from a list.

Video-Chat has made it easier for distance students to work in pairs or do group work. It has rooms designated for just this purpose. Students can freely use these rooms anytime they wish. They simply navigate to the Video-Chat page in Video CMS and select the room they want to join from a list. Tutoring can also be done via Video-Chat. Students don't need to travel to the university. They can book a time that is convenient for them and join Video-Chat from anywhere.

LimeSurvey Module makes it possible to create surveys in the environment. It is a well-known open source application supporting 28 different question types, and its user interface has been translated to over 80 languages. It is quite easy to create web-based questionnaires with its design tools. We use an API to exchange data between the two systems; survey data is saved in a separate database. The user however won't notice that he/she is using another system when filling in a questionnaire in the LimeSurvey application.

Learning Styles Module (LS Module) uses LimeSurvey. The students can fill out a Learning Style survey developed for the Felder-Silverman model [3]. The questionnaire has 44 questions with two alternatives. After completing the questionnaire, the student is taken to the student's personal user profile page. The result of the survey is shown in a graphical form. The diagram consists of four learning style axes. The student's score is shown on each axis. A short explanation and a more detailed explanation of the Felder-Silverman model are available via links that are included in the LS Module. There is also a link which enables the student to compare his/her result with the results of other students. The module also has a lecturer's view. The lecturer is able examine the results of the students for the courses he/she is teaching. Individual test results are not available to the lecturer, as only diagrams of the scores are shown. Information about the learning style model in general is shown to the lecturer as are suggestions for paying attention to different learning styles in teaching and links to additional information. More detailed information can be found in [4].

SUS Survey Module is another module which uses the LimeSurvey application, and its survey is based on the System Usability Scale [5]. The survey consists of ten questions, and it is very easy and quick to complete. The module was mainly designed for getting feedback from students about new modules of the environment and other applications developed for students. The first evaluation of user satisfaction of new pilot modules has typically been carried out with the SUS Survey.

Student Participation Data Module collects participation data from both face-to-face and video participants. Data on the participation of distance learners has been logged since we started using the CiNetCampus Studies environment. Participation of face-to-face students had earlier been tracked by only course-specific attendance sheets. The first version of the module, which collects also face-toface student participation data, was developed as a student project. After the project, some changes were made to it, and it was integrated into the environment. The module is running in iPad kiosk mode in every classroom. A list of all students in the course along with their pictures is displayed on the screen. When attending a face-to-face lecture, the student selects his/her name from the list of course participants on the iPad. There is also a text-based search box where the student's name can be entered in case he/she has forgotten to enroll into the course in LMS. Participation data is then logged into database.

User Support Module is also included in the environment. If users are experiencing problems with viewing, there is a support form they can use to contact technical support. When a support request is filled in, the support personnel also automatically get information regarding the setup of the client's computer. The support staff also gets information about other students watching the same video when the support request was made. This can help in finding out whether the problem affects other students as well. 


\section{ARCHITECTURE OF CINETCAMPUS STUDIES}

The architecture of CiNetCampus Studies is illustrated in Fig.2. The main elements are LMS and Video CMS. Video CMS is the main platform for the modules. LimeSurvey is connected via API to support the survey modules of Video CMS, LS Module and SUS Survey Module.

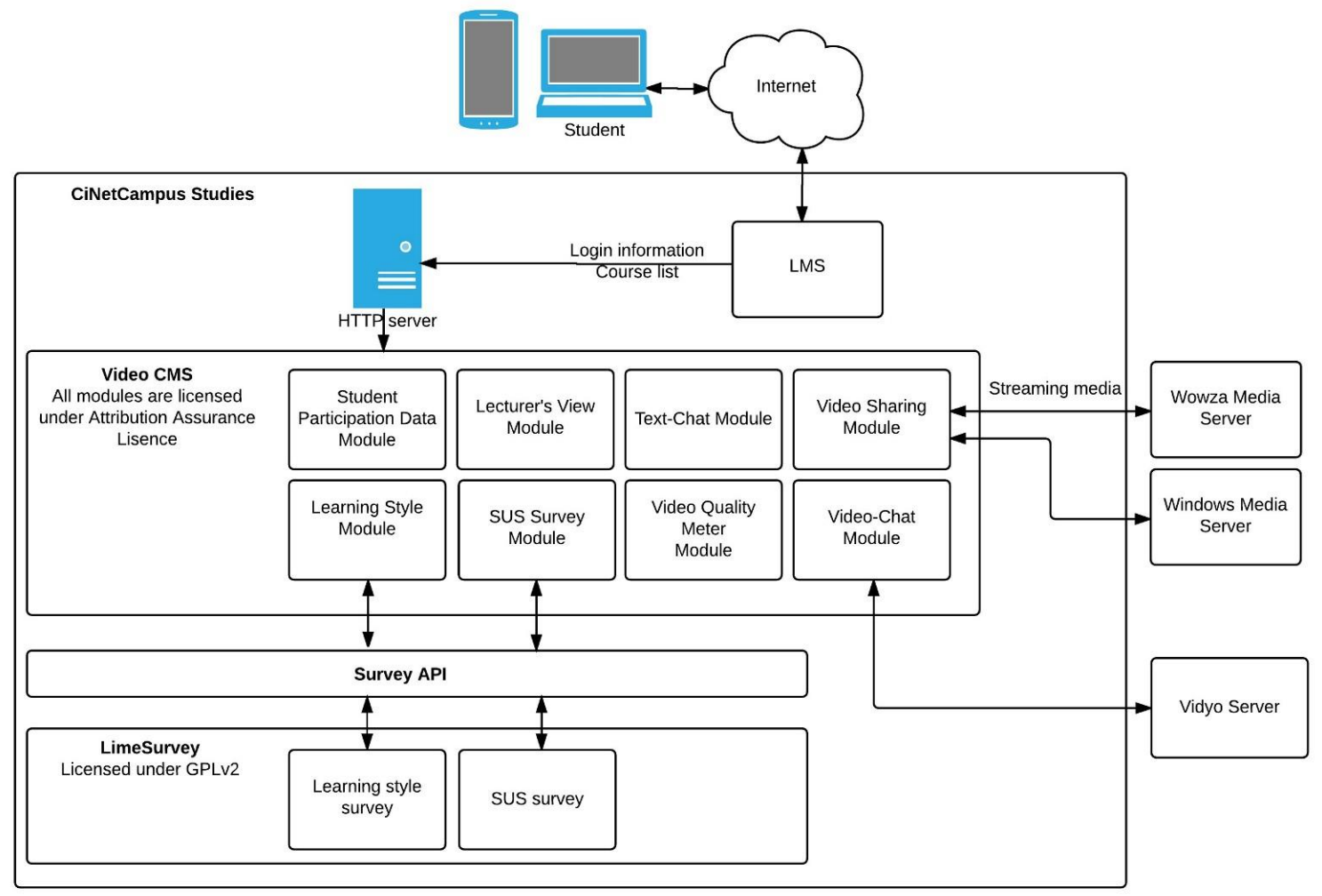

Figure 2. The architecture of CiNetCampus Studies

Video CMS is built on 3-tier architecture, as illustrated in Fig. 3. This means that software is split into three parts. User interface (presentation tier), functional process logic (application tier) and data storage (data tier) are separated. Web browser functions as the presentation tier. The presentation tier sends a request to the application tier. This tier is located on a web server (Apache HTTP Server). The application layer is coded with PHP programming language. The application tier communicates with the data tier, making changes or retrieving information based on the presentation tier's requests. MySQL database functions as the data storage.

Presentation logic uses a template engine called Smarty. Templates can be changed, and we also have changed them. Layout changes can be done easily by modifying the presentation tier. The original presentation tier of ClipBucket is no longer in use, and we have replaced it with our own version.

Even though user management is handled in the LMS, the Video CMS naturally still has to have its own user table. Every user has a unique Video CMS link in the LMS. This link includes the username and a list of course codes the user is a member of. When clicking this link, the user is redirected to the Video CMS login script. The user is granted access to the Video CMS if the security checks based on a shared key known by both systems are passed. After that the user is navigated to the front page of the Video CMS. The user is then able to access only his/her courses' video listings. 


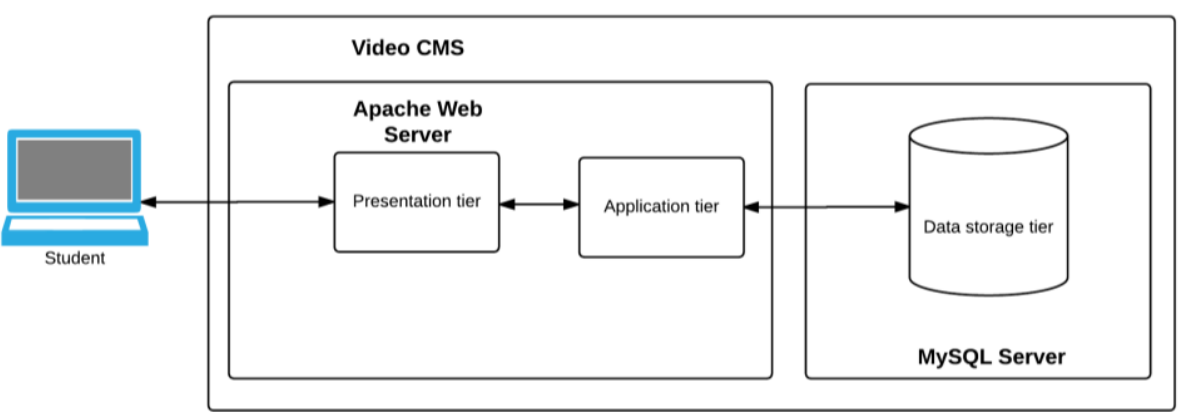

Figure 3. Three-tier architecture

The introduction of Student Participation Data Module required that we needed to update the course member table of the Video CMS more often. The module displays a list of students of the course on the screen of an iPad. That list needs to be up to date so that the students can easily mark themselves as attending the lecture. If a student hasn't logged on to the Video CMS for a while, the system might not have his/her up-to-date course list. It was decided that LMS submits the course list along with their member information once a day. This transfer now happens nightly and also includes course descriptions, which are shown on the course-specific video listing pages of the Video CMS.

Every video in Video CMS has its own record in the database. It contains, among other things, the title of the video, its duration and the video embed codes for all the platforms we support. Video publishing process is carried out with the help of a scheduler tool. Every week, details such as course code along with start and end times of each lecture of the week are entered into the scheduler tool. The scheduler then adds the records for those lectures in the Video CMS database and creates scheduled tasks for publishing and unpublishing of videos.

When a user selects a video from a video list, the video viewing page opens. Currently we support the following platforms: Windows, Linux, Mac, iOS, and Android. The default media player we use is the Flash-based Flowplayer. Flowplayer is an open source media player, which has been modified to work with our Video Quality Meter. It is embedded into the video viewing page. Wowza Media Server is the server where all H.264 encoded video is streamed from. Windows users can also choose to watch videos using the Windows media player. In that case, videos are streamed from a Windows Media Server.

Text-Chat module is another project using an open source code. The original chat only supported one chatroom, but when we integrated the chat to the Video CMS we made some changes. Now every video has its own chatroom. When a user sends a message, it is only visible to those watching the same video. Because videos are available for the duration of a course, it doesn't make sense to leave old chat messages visible. Messages are archived nightly. Lecturer's view uses the same chat module to communicate with the students watching the video.

Video-Chat Module uses a commercial VidyoWeb application. Video-Chat rooms are located on a VidyoPortal server. The rooms used for tutoring and supervising are always reserved for that purpose. Every classroom has its own Video-Chat room. The rooms are named after classrooms. When a lecture is in progress in a classroom, the Video-Chat room of that classroom is reserved for lecture use only. In other times, those rooms can be used for other purposes as well. When a lecturer initiates a Video-Chat a link, Video-Chat is activated to distance learners. When a user selects a room he/she wants to enter and clicks the join-room button, he/she is directed to the VidyoWeb login page located in our own server. The login page detects if the client computer has the VidyoWeb browser plugin installed. If it is not installed, a link where the plugin can be downloaded from is displayed along with installation instructions. The installation takes only a few seconds, and the login process continues immediately after that. The login page then redirects to a Vidyo server with the username of the user and the name of the room he/she wants to join. After that the Video-Chat view is opened. The layout of Video-Chat is built with HTML, and thus it is customizable. The buttons on the page are VidyoWeb API calls, which control the plugin. We have modified the page to make it automatically redirect back to the Video CMS when the user wants to exit the Video-Chat, and disconnect button is pushed.

LimeSurvey Module is the only open source module which isn't licensed under the Attribution Assurance license (AAL). LimeSurvey is used in conjunction with the Learning Style- and SUSmodules, but it isn't really integrated into Video CMS. This is because LimeSurvey is licensed under 
General Public License v2 (GPL), which is classified as a copyleft license. This means that if we integrated LimeSurvey to Video CMS we would have to license the whole system under GPL. Licensing under GPL is not an option for us because of the many limitations set by this license. AAL is a permissive license and suits our needs well. LimeSurvey is installed on the same server as Video CMS. It is used in both Learning Styles and SUS modules. It has been developed with the PHP programming language. It uses MySQL database as its data storage. When logging onto the Video CMS for the first time, the user's username and userid are saved to the LimeSurvey users table. This is done via an API, which we have developed for LimeSurvey. A random string token is also generated. The token is a way of identifying users when they take a survey. Once the user has been created, the API returns the token to the Video CMS. The token is then saved to the record of the user in the Video CMS database.

We are able to keep track of students who have completed surveys. Once a survey has been completed, the student is redirected back to the Video CMS by using a parameter which tells which survey has been completed. When we need the students to answer to a SUS or Learning Style survey, we check if the logged-in student has completed the survey. If not, a notification is displayed. This notification contains a link to the survey with the student's LimeSurvey token as a parameter. Once a student has completed the Learning Style survey he is redirected to his/her personal profile page in the Video CMS. Graphs based on the answers of the user are shown to the student, but since they are located in the LimeSurvey database we can't access them directly. A request is sent to the $\mathrm{API}$, and the results are returned to the page and used as the basis of the chart.

\section{THE ROLE OF CINETCAMPUS STUDIES IN OUR EDUCATION}

Our students of Master's degree in Mathematical Information Technology are all working adult students whose possibilities to participate regularly in face-to-face teaching are very limited. Students' need for adaptive solutions to participate in education has been the primary motivation for the educational model. The development work on our blended educational model has been going on over a decade. In the beginning, our carrying thought was to make participation in education as flexible as possible as well as to give more choices for different kind of learners.

The CiNetCampus Studies environment can be seen as a technical realization of our blended educational model. The environment enables the students to participate in education in many different ways by flexibly combining various alternatives. In the developing process, various distance education and other technological solutions have played remarkable roles. The technologies available typically define how far the development of the model can be carried to. However, the practices formed around the chosen technologies have shaped the final realization of the educational model.

Our current blended educational model is based on face-to-face teaching and strong utilization of video lectures. All lectures are given in face-to-face sessions, which are recorded in live teaching situations and distributed, both live and as on-demand videos. Face-to-face teaching is arranged as very compact entities, mainly for Friday afternoons and Saturdays. In practice, this means that the duration of a single lecture session equals 3-4 traditional lectures. The aim of the scheduling is to give working students realistic opportunities to participate in face-to-face teaching within the time constraints.

It is difficult to find a commercial video production system that would, comprehensively and without compromises, meet our needs. For that reason, we have build own almost fully automated production system by utilizing, combining and complementing various equipment and software solutions available. The videos are automatically produced without any extra personnel from face-to-face teaching situations in a way that the recording is transparent for the students and the lecturer. More detailed information about the production system is available in [5].

Video lecture production makes it possible for the students to participate in study with videos, either from distance, with the help of real time video or by using on-demand videos during times more suitable for them. An important principle in our model is that the students do not need to decide about their participation mode beforehand; they have the freedom to select, for each lecture, the participation mode that is the most suitable for their own particular circumstances at the time. Thus, the student can participate in education in many different ways by flexibly combining various alternatives. A quite unique feature in blended learning is that the student alone defines the degree to which he/she participates in video-aided study. We can say that the degree of blended learning adapts the student's need in our model. 
Since video lectures play important role in our educational model, it is also important to provide solutions for interaction during live and on-demand video lectures. In all participating modes, the students can communicate with each other via synchronized channels in the virtual CiNetCampus Studies environment. As a default, distance students can use text chat to communicate with the lecturer or other students during face-to-face teaching and live video lecture. When necessary, only the lecturer can open a video-chat during the lecture. In other times, also students can use the videochat freely. To support interaction, the students are visible to each other in all participating modes. In face-to-face teaching situations, the images of all live video lecture participants are visible to other participants and, with the help of the Lecturer's View Module, can be seen on the back wall of the teaching area also. Since the participants in face-to-face teaching can logon to CiNetCampus studies environment when entering the lecture room, they will be visible to other students. Moreover, the students watching on-demand video lectures at the same time are visible to each other. In a lecture situation, it is important for the lecturer that the presence of the distance students be made visible in order to give them their share of attention. It is also advisable that the students see each other when carrying out joint practices.

Improved access to education was one of the primary motivations when we started to develop our educational model and its technical realization. When making educational arrangements for adult students, who have many responsibilities in addition to family, hobbies and work, the emphasis will be on reducing scheduling-related conflicts. Strong utilization of video lectures in our model clearly increases time- and location-independence of the education and in that way decreases schedulingrelated conflicts. The student can pace his/her study in the way desired and when it seems convenient. The model also supports revision. On-demand videos can be used for revision, regardless of the initial way of participation. Besides giving support to accessibility and revision, the model's provision of various participation modes enables support to students with different study preferences.

\section{CONCLUSION AND FUTURE WORK}

Providing lecture videos in higher education has multiple benefits for students but poses many challenges for the education organizer. This paper presents a technical platform with practices for blended learning environment which is based on face-to-face learning and strong utilization of video lectures. The model can be applied in any educational organization that utilizes streaming videos in their educational practices.

The core of our virtual campus, CiNetCampus Studies, consists of a learning management system (LMS) and a video content management system (Video CMS). In addition to services typical for LMS, it has a significant function to provide centralized management of videos and synchronized interaction channels during video lectures. Since every organization has its own changing requirements, the virtual campus environments should be adaptable. For this reason, our CiNetCampus Studies is modular. The Video CMS works also as a main platform for new modules. Currently, our versatile module collection provides services for video lectures and helps us to develop our education in line with our pedagogical purposes to benefit our students. The goal of the future development is a highly personalized learning environment.

\section{ACKNOWLEDGMENTS}

The research for this paper was financially supported by the European Social Fund, grant no. S20073, without which the present study could not have been completed. The authors wish to thank the Central Finland Centre for Economic Development, Transport and the Environment for their help.

\section{REFERENCES}

[1] Bonk, C.J., and Graham, C.R. (2006). Handbook of Blended Learning: Global Perspectives. Local Designs, San Francisco, CA:Pfeiffer Publishing.

[2] Dziuban, C.D., Hartman, J.L., and Moskal, P.D. (2004). Blended Learning. Educause Center for Applied Research Bulletin (7), pp. 1-12.

[3] Felder, R., and Solomon, B., Index of Learning Styles (ILS). http://www4.ncsu.edu/unity/lockers/users//felder/public/lLSpage.html 
[4] Hakala, I., Härmänmaa, T., and Laine, S. (2016). Learning Styles Module as a Part of a Virtual Campus. In Proceedings of IEEE Global Engineering Education Conference, Abu Dhabi, United Arab Emirates.

[5] Brooke, J. (1996). SUS: a ‘Quick and Dirty’ Usability Scale, In Usability Evaluation in Industry, P.W. Jordan, B. Thomas, B.A. Weerdmeester, and I.L. McClelland (Eds.), London, Taylor and Francis, pp. 189-194. 